\title{
High Frequency Interaction-Induced Anisotropic Rototranslational Light Scattering Spectra of Gaseous Carbon Dioxide
}

\author{
M.S.A. El-KADER ${ }^{a, *}$, T. BANCEWICZ ${ }^{b}$ AND G. MAROUlis ${ }^{c}$ \\ ${ }^{a}$ Department of Engineering Mathematics and Physics, Faculty of Engineering, Giza, 12211, Egypt \\ ${ }^{b}$ Nonlinear Optics Division, Institute of Physics, Adam Mickiewicz University \\ Grunwaldzka 6, 60-780 Poznań, Poland \\ ${ }^{c}$ Department of Chemistry, University of Patras, Caratheodory St, GR-26500 Patras, Greece
}

(Received August 9, 2010; in final form January 7, 2011)

\begin{abstract}
The anisotropic rototranslational scattering of carbon dioxide gas is studied theoretically at $294.5 \mathrm{~K}$, in the frequency range $10-470 \mathrm{~cm}^{-1}$, at a density of $1.026254 \mathrm{~mole} / \mathrm{litre}$. The anisotropic double differential cross-section for scattered light is calculated theoretically using new site-site Morse-Morse-Morse-spline-van der Waals intermolecular potentials with the parameters fitted to the different thermophysical and transport properties. Our theoretical calculations take into account multipole contributions from the first- and second-order dipole-induced dipole, first-order dipole-induced octopole and first-order dipole-dipole-quadrupole light scattering mechanisms as well as their cross contributions. The irreducible spherical form for the induced operator of these light scattering mechanisms was determined. The high frequency wings are discussed in terms of the collision-induced rotational Rayleigh effect and estimates for the dipole-octopole polarizability $E_{4}$, is obtained and checked with the $a b$ initio theoretical value. Good agreement is obtained at moderate frequencies between the theoretical and experimental spectra. When an exponential contribution $\exp \left(-\nu / \nu_{0}\right)$, with $\nu_{0}=115 \mathrm{~cm}^{-1}$ is considered to model very short-range light scattering mechanism, good agreement is found over the whole frequency range.
\end{abstract}

PACS: 78.47.je, 34.20.Gj

\section{Introduction}

Collision-induced light scattering spectra have commonly been used to study such properties as intermolecular interaction potentials, collision induced dipoles, and polarizabilities. For instance, the scattered intensity in the high frequency spectral region has been used to extract information about some of the molecular dipolemultipole polarizability tensor components of several isotropic molecules such as $\mathrm{CH}_{4}, \mathrm{CF}_{4}, \mathrm{CCl}_{4}$ and $\mathrm{SF}_{6}$ [1-5] and of anisotropic molecules belonging to symmetry group $C_{2 v}$ or $D_{\infty h}\left(\mathrm{~N}_{2}\right)[6,7]$. The special case of very light molecules $\mathrm{H}_{2}$ and $\mathrm{D}_{2}$ has also been investigated [8-12]. Such a procedure is correct because in this case all the scattered intensity is due to collision-induced polarizabilities that arise from long range interaction mechanisms involving high order multipole polarizabilities.

However, for any system under consideration, one must

* corresponding author; e-mail:

mohamedsayedabdelkader@yahoo.com make sure that the experimental conditions, and/or the theory used for their analysis, are suitable for the extraction of the desired information. In this paper we focus on the far wings of the anisotropic rototranslational scattering spectra from gaseous carbon dioxide, which differs from those of tetrahedral and octahedral molecules because of the presence of an anisotropic polarizability which is responsible for an allowed rotational spectrum. Such a spectrum adds to the collision-induced one and the problem arises about their separability.

In this work, accurate measurements of the anisotropic light scattering intensities for $\mathrm{CO}_{2}$ gas from a few $\mathrm{cm}^{-1}$, up to $470 \mathrm{~cm}^{-1}$ with a higher resolution, have been added to study more closely the low-frequency part of the spectra [13]. Such a spectrum, recorded at room temperature $294.5 \mathrm{~K}$, will be analyzed in order to develop refined models of the relevant interaction-induced properties, which permit an accurate reproduction of the observed spectra from lineshapes calculations.

To compare our theoretical spectra with the experimental ones, we calculate these spectra taking into consideration the first- and second-order dipole-induced dipole light scattering mechanisms as well as the dipole- 
induced octopole effect related with the nonuniformity of the local field acting on the $\mathrm{CO}_{2}$ molecule. The irreducible spherical components of the second-order dipoleinduced dipole (DID), dipole-induced octopole (DIO) and hyperpolarizability-permanent quadrupole $\mathrm{B} \Theta$ light scattering mechanisms are considered.

Our paper is organized as follows: the intermolecular potential form adopted is presented in Sect. 2. Calculations of the thermodynamic and transport properties to determine the different potential parameters is detailed in Sect. 3. The light scattering experiments are briefly described in Sect. 4. In Sect. 5 we give the multipolar theory of the spectral shape of the scattered light, devoting special attention to main broadening procedures of the rotational stick spectrum due to translational motions of the $\mathrm{CO}_{2}$ molecules. In Sect. 6 we compare the experimentally measured spectra with those obtained theoretically; whereas in Sect. 7 a summary is given.

\section{Multi-property analysis and the intermolecular potential}

In order to calculate the line profiles, the intermolecular potential is needed. Results with different potential parameters can be compared with experiment to assess the quality of the potential.

The intermolecular potential we use is an MorseMorse-Morse-spline--van der Waals (M3SV) generalization of the Morse-spline-van der Waals (MSV) form [14]. In regions $i=\mathrm{I}$, II, and III it is represented by the Morse potentials

$$
\begin{aligned}
& V_{i}(r)=\varepsilon\left[\exp \left(-2 \xi_{i}\left(r / r_{m}-1\right)\right)\right. \\
& \left.-2 \exp \left(-\xi_{i}\left(r / r_{m}-1\right)\right)\right] .
\end{aligned}
$$

These Morse potentials are chosen so that they are continuous and have continuous first derivatives where they join.

The potential $V$ is represented in region IV as a single cubic Hermite spline

$$
\begin{aligned}
& V_{\mathrm{IV}}=\varepsilon\left\{\beta_{1}+\left(x-x_{1}\right)\left[\beta_{2}+\left(x-x_{2}\right)\right.\right. \\
& \left.\left.\quad \times\left(\beta_{3}+\left(x-x_{1}\right) \beta_{4}\right)\right]\right\},
\end{aligned}
$$

where $x=r / r_{m}$ is a reduced distance, and in region $\mathrm{V}$ by the asymptotic London or van der Waals expansion

$$
V_{\mathrm{V}}=\varepsilon\left(-C_{6} x^{-6}-C_{8} x^{-8}-C_{10} x^{-10}\right),
$$

where $C_{6}, C_{8}$, and $C_{10}$ are fitting parameters.

Given $V_{\text {III }}$ and $V_{\mathrm{V}}, V_{\mathrm{IV}}$ is completely determined by the requirements of continuity of the potential and its slope.

Even at the present M3SV level, there are ten free parameters $\varepsilon, \xi_{\mathrm{I}}, \xi_{\mathrm{II}}, \xi_{\mathrm{III}}, r_{m}, x_{1}, x_{2}, C_{6}, C_{8}$, and $C_{10}$ which are far too many to determine from the present data. Accordingly we proceeded as follows: dispersion force constants $C_{6}, C_{8}$ and $C_{10}$ were fixed at the values given by Visser and Wormer [15]. Similarly, $x_{1}$ is usually taken as the inflection point of $V_{\mathrm{III}}$,

$$
x_{1}=r_{1} / r_{m}=\left[1+\ln 2 / \xi_{\mathrm{III}}\right]
$$

and $x_{2}$ is usually held fixed at some value $(\approx 1.76)$ such that $r_{2}=r_{m} x_{2}$ is large enough that the series in Eq. (3) is expected to be adequate but small enough to prevent the spline in Eq. (2) from oscillating. Furthermore, in the initial fitting the parameter $\xi_{\mathrm{I}}=\xi_{\mathrm{II}}=\xi_{\mathrm{III}}=\xi(\mathrm{MSV}$ potential) was held fixed at some values while $\varepsilon$ and $r_{m}$ were chosen to minimize $\delta_{\mathrm{B}}, \delta_{\eta}, \delta_{\mathrm{D}}$ and $\delta_{\text {Iso }}$, the rms deviations calculated from the pressure second virial coefficient, viscosity, self diffusion coefficient and isotopic thermal factor. This minimization is further supported by calculating $\delta_{\lambda}$ the rms deviations calculated from the experimental thermal conductivity. This decision leads

\begin{tabular}{|c|c|c|c|c|c|}
\hline $\begin{array}{r}\text { Potential } \\
\text { Parameters }\end{array}$ & 1 & 2 & 3 & 4 & 5 \\
\hline$\varepsilon[\mathrm{K}] / k_{\mathrm{B}}$ & 244.6 & 245.8 & 247.5 & 249.3 & 250.8 \\
\hline$\sigma[\AA]$ & 3.768 & 3.762 & 3.759 & 3.754 & 3.75 \\
\hline$r_{m}[\AA]$ & 4.285 & 4.27 & 4.25 & 4.23 & 4.215 \\
\hline$\xi_{\mathrm{I}}$ & 6.65 & 6.8 & 7.0 & 7.15 & 7.2 \\
\hline$\xi_{\text {II }}$ & 5.74 & 5.825 & 6.0 & 6.16 & 6.28 \\
\hline$\xi_{\text {III }}$ & 4.42 & 4.45 & 4.5 & 4.55 & 4.6 \\
\hline$r_{1}[\AA]$ & 4.957 & 4.935 & 4.905 & 4.874 & 4.8501 \\
\hline$r_{2}[\AA]$ & 7.285 & 7.0455 & 6.80 & 6.726 & 6.6397 \\
\hline$\beta_{1}$ & -0.75 & -0.75 & -0.75 & -0.75 & -0.75 \\
\hline$\beta_{2}$ & 1.316 & 1.429 & 1.5585 & 1.58 & 1.6017 \\
\hline$\beta_{3}$ & -1.646 & -1.61 & -1.5506 & -1.59 & -1.6265 \\
\hline$\beta_{4}$ & -0.967 & -1.8733 & -3.207 & -3.326 & -3.4674 \\
\hline$C_{6}$ & 0.644 & 0.6547 & 0.6688 & 0.683 & 0.6935 \\
\hline$C_{8}$ & 0.478 & 0.4897 & 0.5049 & 0.521 & 0.5323 \\
\hline$C_{10}$ & 0.3425 & 0.353 & 0.3674 & 0.3824 & 0.3939 \\
\hline$\delta_{\mathrm{B}}$ & 0.97 & 0.93 & 0.88 & 0.825 & 0.92 \\
\hline$\delta_{\eta}$ & 0.85 & 0.62 & 0.53 & 0.81 & 0.6 \\
\hline$\delta_{\mathrm{D}}$ & 0.83 & 0.55 & 0.44 & 0.57 & 0.63 \\
\hline$\delta_{\text {Iso }}$ & 0.9 & 0.65 & 0.51 & 0.56 & 0.91 \\
\hline$\delta_{\lambda}$ & 0.74 & 0.68 & 0.62 & 0.84 & 0.94 \\
\hline$\delta_{\mathrm{t}}$ & 0.91 & 0.77 & 0.69 & 0.76 & 0.83 \\
\hline \multicolumn{6}{|c|}{$\begin{array}{l}\delta^{a} \text { is defined by } \delta^{a}=\sqrt{(1 / N) \sum_{j=1}^{N}\left(1 / n_{J} \sum_{j=1}^{n_{j}} \Delta_{j i}^{-2}\left(P_{j i}-p_{j i}\right)^{2}\right)} \\
\text { where } P_{j i} \text { and } p_{j i} \text { are, respectively, the calculated and experi- } \\
\text { mental values of property } j \text { at point } i \text { and } \Delta_{j i} \text { is the experimental } \\
\text { uncertainty of property } j \text { at point } i \text {. The subscripts } \mathrm{B}, \eta, \mathrm{D} \text {, } \\
\text { Iso, } \lambda \text { and t refer, respectively, to the pressure second virial } \\
\text { coefficient, the viscosity, the self diffusion coefficient, the isotopic } \\
\text { thermal factor, the thermal conductivity and total. }\end{array}$} \\
\hline
\end{tabular}
to potential 3 of Table I as our best estimate of the $\mathrm{CO}_{2}-\mathrm{CO}_{2}$ intermolecular potential.

TABLE I

Parameters of the trial potentials and the associated values of $\delta^{a}$.

\section{Calculations}

In this section the calculations of the experimental quantities are described. The Monchick-Mason approximation [16] which neglects the effect of inelastic transitions on the relative kinetic energy is applied in the computations of the transport properties.

\subsection{Analysis of pressure second virial coefficients}

An effective mean for checking the validity of the different potential parameters can be made using pressure 
second virial coefficient data $[17,18]$ at different temperatures. The pressure second virial coefficient $B$ at temperature $T$ was calculated classically from [19]:

$$
B(T)=2 \pi N_{0} \int_{0}^{\infty}\left[1-\exp \left(-V(r) / k_{\mathrm{B}} T\right)\right] r^{2} \mathrm{~d} r,
$$

where $N_{0}$ is the Avogadro number. The calculated $B$ were compared with the experimental results of Lemmon et al. [17] and with the literature ones of Boushehri et al. [18]. The uncertainty in the data was taken as $0.5 \%$.

\subsection{Analysis of traditional transport properties}

An additional check on the proposed potential consists of the calculation of the transport properties i.e. viscosity $\eta(T)$, diffusion coefficient $D(T)$, isotopic thermal factor Iso $(T)$ and thermal conductivity $\lambda(T)$ at different temperatures of carbon dioxide, obtained via the formulae of Monchick et al. [16] and their comparison to the accurate experimental results of Lemmon and McLinden [17] and to the literature ones of Boushehri et al. [18] and Vesovic et al. [20].

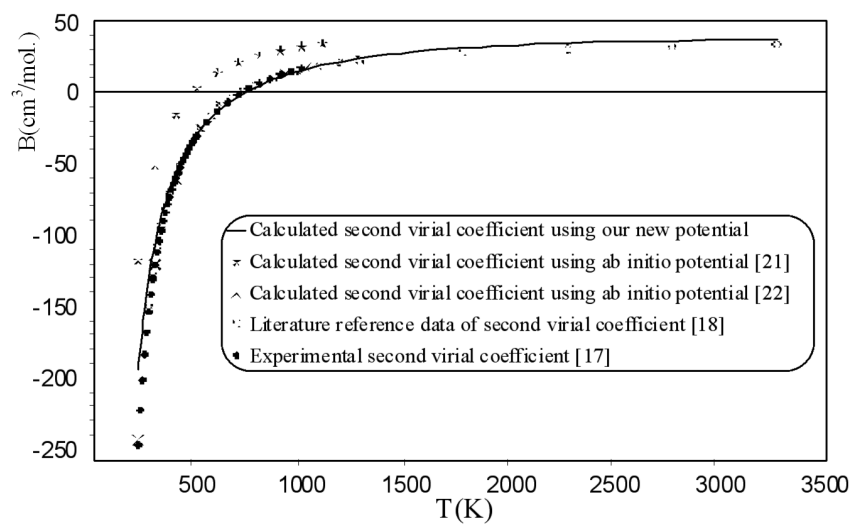

Fig. 1. $\mathrm{CO}_{2}-\mathrm{CO}_{2}$ pressure second virial coefficients in $\mathrm{cm}^{3} \mathrm{~mol}^{-1}$ versus temperature in K. The calculations were performed using two different ab initio potentials $[21,22]$ and our new M3SV potential given in Table I and labeled 3.

Results of the classical pressure second virial coefficients $B(T)$ calculations with the quantum corrections performed with our new M3SV potential and other $a b$ initio potentials $[21,22]$ considered in this work are presented in Fig. 1. As it may be clearly seen, our potential gives the best agreement with the experimental values over a high range of temperatures.

Also, the comparison between the calculations using our new potential and experiment is shown in Fig. 2 for the thermal conductivity $\lambda(T)$, isotopic thermal factor Iso $(T)$, self diffusion coefficient $D(T)$ and viscosity $\eta(T)$. The agreements are excellent in the whole temperature range.

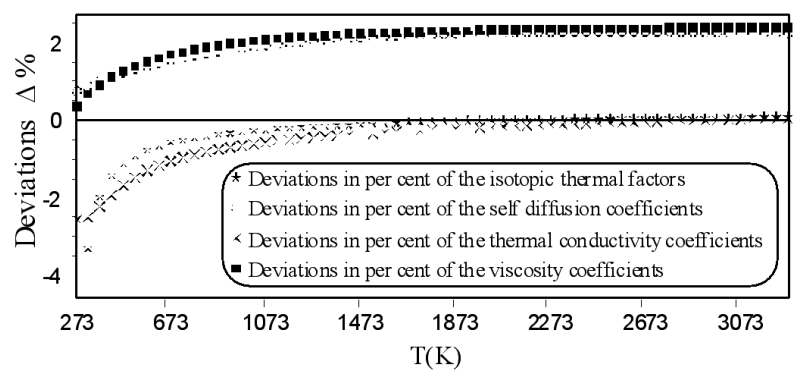

Fig. 2. Fig.2. Deviations in per cent as a function of temperature of the viscosity coefficients the self diffusion coefficients $D(T)$, isotopic thermal factor Iso $(T)$ and the thermal conductivity coefficients from the experimental results. Calculations were performed using M3SV potential given in Table I and labeled 3.

\section{Light scattering spectrum}

The measurement and calculation of the $\mathrm{CO}_{2}-\mathrm{CO}_{2}$ light scattering spectra have been fully reported elsewhere [7, 13]. For the sake of completeness, some details are repeated here. Collision-induced light scattering refers to scattering from clusters (here pairs) of interacting anisotropic molecules. The spectral feature of interest is a broad depolarized band about the Rayleigh line. The experimental setup is that typical of laser Raman spectroscopy. Excitation radiation was provided by an argon-ion laser operating at $514.5 \mathrm{~nm}$ at a power of $2 \mathrm{~W}$. The polarizations of the laser beam are perpendicular and parallel to the scattering plane, that is the plane defined by the incident and the scattered beams. The intensities of the scattered light are then referred to as $I_{\perp}(\nu)$ and $I_{\|}(\nu)$ and constitute the polarized (which is related to the isotropic spectrum) and depolarized (anisotropic) spectrum, respectively. Intensities were put on an absolute scale by taking as reference a rotational line of $\mathrm{H}_{2}$. The anisotropic light scattering spectrum of gaseous carbon dioxide in absolute units at room temperature is shown in Fig. 3 [13]. The uncertainty in the scattering data was taken as $10 \%$.

\section{Theory}

For linear centrosymmetric molecules such as $\mathrm{CO}_{2}$ we encounter more complicated conditions for observation of collision-induced scattering (CIS) than in the case of globular molecules. The polarizability tensor of linear molecules is no longer isotropic and its anisotropy leads to a very strong allowed rotational spectrum. As a result of a pressure broadening mechanism, this allowed spectrum produces substantial intensities even at very high frequencies $[23,24]$. The excess interaction-induced part of the pair polarizability creates a continuous rototranslational relatively weak but very broad spectral distribution of scattered light. Finally in experiment, both the allowed spectrum and CIS spectrum, overlap. 


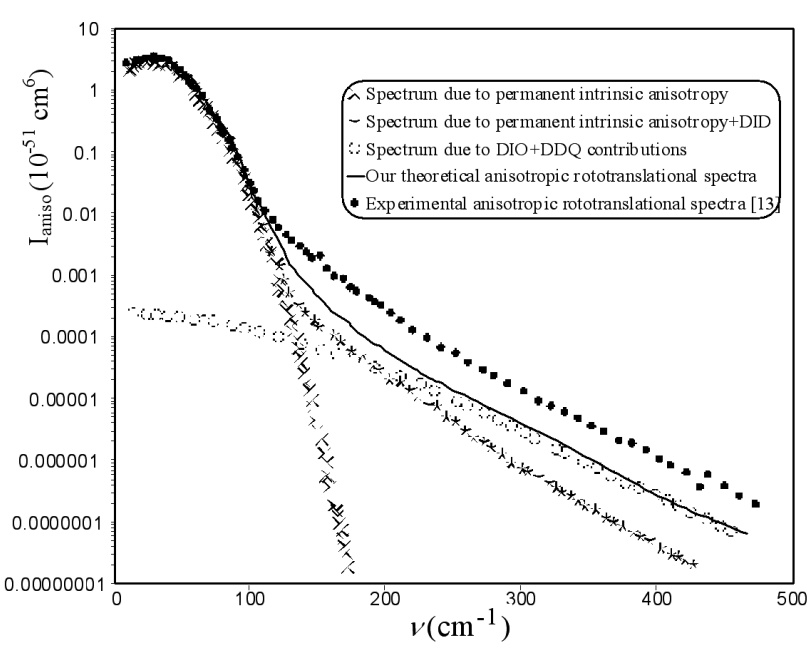

Fig. 3. The intensity of the rototranslational anisotropic spectra $\left(E_{4}=1.967 \AA^{5}\right)$ of $\mathrm{CO}_{2}$ at $294.5 \mathrm{~K}$ as a function of frequency shifts. The points refer to the experiment presented in [13]. Calculations were performed using M3SV potential with the parameters given in Table I and labeled 3.

For anisotropic spectra, collision-induced contribution appears to be significant only at very high frequencies.

Consider scattering of light linearly polarized in the scattering plane and detected with no analyzer. Then the double differential cross-section of the scattered light reads

$$
\left(\frac{\partial^{2} \sigma}{\partial \Omega \partial \omega}\right)_{H}=\frac{1}{5} K_{i} K_{s}^{3} \frac{1}{2 \pi} \int \exp (-\mathrm{i} \omega t) F_{22}^{0}(t) \mathrm{d} t,
$$

where

$$
F_{22}^{0}(t)=\left\langle\boldsymbol{A}^{(2)}(0) \odot \boldsymbol{A}^{(2)}(t)\right\rangle
$$

is the autocorrelation function of the irreducible second rank pair polarizability tensor $\boldsymbol{A}^{(2)}$ of the scattering molecules. In Eq. (7), $\odot$ denotes a scalar tensor product and \langle\rangle is a canonical average.

The pair polarizability tensor $\boldsymbol{A}^{(2)}$ is composed of two parts

$$
\boldsymbol{A}^{(2)}={ }_{(1)} \boldsymbol{A}^{(2)}+{ }_{(2)} \boldsymbol{A}^{(2)}+\Delta \boldsymbol{A}^{(2)},
$$

the permanent pair polarizability (1) $A^{(2)}+{ }_{(2)} A^{(2)}$ and the excess pair polarizability $\Delta \boldsymbol{A}^{(2)}$, originating in the intermolecular interactions. The pair induction operator $\Delta \boldsymbol{A}^{(2)}$ is dependent on the molecular orientations $\Omega_{1}$ and $\Omega_{2}$, and the relative molecular separation $r$; for linear molecules $\Delta \boldsymbol{A}^{(2)}$ has the form of an expansion in spherical harmonics $Y_{m}^{l}(\Omega)$ :

$$
\begin{aligned}
& \Delta \boldsymbol{A}_{\mu}^{(q)}=\frac{(4 \pi)^{3 / 2}}{(2 q+1)^{1 / 2}} \sum_{\lambda_{1}, \lambda_{2}, \Lambda, L} \boldsymbol{B}_{\lambda_{1}, \lambda_{2}, \Lambda, L}^{(q)}(r) \\
& \quad \times\left(\boldsymbol{Y}^{(\mathrm{L})}(\hat{r}) \otimes\left[\boldsymbol{Y}^{\left(\lambda_{1}\right)}\left(\Omega_{1}\right) \otimes \boldsymbol{Y}^{\left(\lambda_{2}\right)}\left(\Omega_{2}\right)\right]^{(\Lambda)}\right)_{\mu}^{(q)} .
\end{aligned}
$$

In the range of intermolecular separations $r$ where the molecular charge distributions do not overlap, the induced variation $\boldsymbol{\Delta} \boldsymbol{A}^{(2)}$ in the polarizability, in Cartesian tensor notation, up to order $r^{-6}$, reads [23-25]:

$$
\begin{aligned}
& \Delta \boldsymbol{A}_{\alpha \beta}=\left(1+P^{12}\right)\left[\boldsymbol{A}_{\alpha \gamma}^{(1)} \boldsymbol{T}_{\gamma \delta}(r) \boldsymbol{A}_{\delta \beta}^{(2)}\right. \\
& \quad+\boldsymbol{A}_{\alpha \gamma}^{(1)} \boldsymbol{T}_{\gamma \delta}(r) \boldsymbol{A}_{\delta \varepsilon}^{(2)} \boldsymbol{T}_{\varepsilon \phi}(r) \boldsymbol{A}_{\phi \beta}^{(1)} \\
& \quad+\frac{1}{15} \boldsymbol{A}_{\alpha \gamma}^{(1)} \boldsymbol{T}_{\gamma \delta \varepsilon \phi}(r) \boldsymbol{E}_{\beta, \delta \varepsilon \phi}^{(2)}+\frac{1}{15} \boldsymbol{E}_{\alpha, \gamma \delta \varepsilon}^{(2)} \boldsymbol{T}_{\gamma \delta \varepsilon \phi}(r) \boldsymbol{A}_{\phi \beta}^{(1)} \\
& \left.\quad-\frac{1}{9} \boldsymbol{B}_{\alpha \beta, \gamma \delta}^{(1)} \boldsymbol{T}_{\gamma \delta, \varepsilon \phi}(r) \boldsymbol{\Theta}_{\varepsilon \phi}^{(2)}+\ldots\right],
\end{aligned}
$$

where $\boldsymbol{A}$ is the dipole-dipole polarizability tensor of the unperturbated molecule, $\boldsymbol{E}$ is the dipole-octopole polarizability tensor, $\boldsymbol{B}$ the dipole-dipole-quadrupole hyperpolarizability tensor, $\boldsymbol{\Theta}$ the permanent quadrupole moment of the molecule, moreover $\boldsymbol{T}_{\alpha_{1} \ldots \alpha_{n}}(r)=$ $\nabla_{\alpha_{1}}, \ldots \nabla_{\alpha_{n}}\left(r^{-1}\right)$, whereas $P^{12}$ permutes the indices 1 and 2. The first and second term of inductional polarizability (10) originates, respectively, in the first-and second-order DID light scattering mechanism. The third and fourth terms are due to the DIO mechanism whereas the last term comes from the permanent quadrupole moment of the molecule and its dipole-dipole-quadrupole hyperpolarizability tensor $\boldsymbol{B}$.

The irreducible spherical tensors version of Eq. (10) has been elaborated elsewhere [6, 26, 27]. The induced polarizability $\Delta \boldsymbol{A}^{(2)}$ is fully defined in spherical tensor if the explicit form of all nonzero $\boldsymbol{B}_{\lambda_{1}, \lambda_{2}, \Lambda, L}^{(q)}(r)$ coefficients of Eq. (9) is available. The explicit form of all nonzero $\boldsymbol{B}$ 's of Eq. (10) are listed in Appendix of Ref. [13].

We calculate the pure rotational and translational parts of our spectrum assuming negligible translational-rotational coupling between $\mathrm{CO}_{2}$ molecules. Then the resulting spectrum takes the form of the convolution of the rotational and translational parts

$$
\frac{\partial^{2} \sigma}{\partial \Omega \partial \omega}=K_{i} K_{s}^{3} \int\left(\frac{\partial^{2} \sigma}{\partial \Omega \partial \omega^{6}}\right)^{\mathrm{rot}}\left(\frac{\partial^{2} \sigma}{\partial \Omega \partial\left(\omega-\omega^{\prime}\right)}\right)^{\mathrm{tr}} \mathrm{d} \omega .
$$

From the above procedure we note that the following molecular parameters:

$$
C_{\lambda_{1}, \lambda_{2}, L}^{(q)}(r)=\sum_{\Lambda}\left[\boldsymbol{B}_{\lambda_{1}, \lambda_{2}, \Lambda, L}^{(q)}(r) \boldsymbol{B}_{\lambda_{1}, \lambda_{2}, \Lambda, L}^{(q)}(r)\right]
$$

govern the interaction-induced rotational branches of our stick spectrum. We calculate these parameters using spherical components of Eq. (9) which are listed in Ref. [13]. Consequently the translational part of our spectrum is described by the following functions:

$$
\begin{aligned}
& { }^{(L, n, k)} S^{\operatorname{tr}}(t)=\frac{4 \pi}{2 L+1}\left\langle r^{-n}(0) \boldsymbol{Y}_{\mathrm{L}}[\hat{r}(0)]\right. \\
& \left.\odot r^{-k}(t) \boldsymbol{Y}_{\mathrm{L}}[\hat{r}(t)]\right\rangle .
\end{aligned}
$$

These functions depend on $L, n$, and $k$. However, since

$$
{ }^{(L, n, k)} S^{\operatorname{tr}}(t)={ }^{(L, k, n)} S^{\operatorname{tr}}(t)
$$

different translational functions emerge only for different pairs of indices $L$ and $n+k$. Finally, 31 rotational stick spectra are computed resulting from different rotational branches: $\Delta J_{1}=0, \pm 2, \pm \lambda_{1} ; \Delta J_{2}=0, \pm 2, \pm \lambda_{2}$ and within each branch from different translational func- 
tions. To deal with translational motion we calculate the Birnbaum-Cohen model spectrum of $(L, n, k) S^{\operatorname{tr}}(t)$ which, by way of the zeroth, second, and fourth translational moments is given by [28]:

$$
\begin{aligned}
& { }^{(L, n, k)} S_{\mathrm{BC}}^{\operatorname{tr}}(\omega)=\frac{M 0(L, n, k) \tau_{1}^{(L, n, k)}}{\pi} \\
& \quad \times \exp \left(\tau_{2}^{(L, n, k)} / \tau_{1}^{(L, n, k)}\right) \\
& \quad \times \exp \left(\omega \tau_{0}\right) \frac{Z^{(L, n, k)} K_{1}\left[Z^{(L, n, k)}\right]}{1+\left(\omega \tau_{1}^{(L, n, k)}\right)^{2}}
\end{aligned}
$$

with the parameters defined as:

$$
\begin{aligned}
& \tau_{0}=\hbar / 2 k_{\mathrm{B}} T \\
& \tau_{1}^{(L, n, k)}=[\hat{M} 2(L, n, k)]^{-1 / 2} \\
& \quad \times\left\{\hat{M} 4(L, n, k) / 3[\hat{M} 2(L, n, k)]^{2}-1\right\}^{1 / 2}, \\
& \quad \tau_{2}^{(L, n, k)}=[\hat{M} 2(L, n, k)]^{-1 / 2} \\
& \quad \times\left\{\hat{M} 4(L, n, k) / 3[\hat{M} 2(L, n, k)]^{2}-1\right\}^{-1 / 2}, \\
& Z^{(L, n, k)}=\frac{\left[1+\left(\omega \tau_{1}^{(L, n, k)}\right)^{2}\right]^{1 / 2}\left[\tau_{0}^{2}+\left(\tau_{2}^{(L, n, k)}\right)^{2}\right]^{1 / 2}}{\tau_{1}^{(L, n, k)}},
\end{aligned}
$$

and $K_{1}\left(Z^{(L, n, k)}\right)$ is a modified Bessel function of the second kind.

We calculate the successive spectral moments for successive $L$ and $n+k$ by means of the following formulae $[27,29]$ :

$$
\begin{aligned}
& M 0(L, n, k)=\left\langle r^{-(n+k)}\right\rangle, \\
& \hat{M} 2(L, n, k)=\left(\beta m_{12}\right)^{-1}\left(L+L^{2}+n k\right) \\
& \quad \times\left\langle r^{-(n+k+2)}\right\rangle / M 0(L, n, k), \\
& \hat{M} 4(L, n, k)=\left(\beta m_{12}\right)^{-2}\left\{\left(2 L+7 k L-k^{2} L+5 L^{2}\right.\right. \\
& \quad+7 k L^{2}-k^{2} L^{2}+6 L^{3}+3 L^{4}+7 k n+k^{2} n+7 L n \\
& \quad+4 k L n+7 L^{2} n+4 k n L^{2}+k n^{2}+3 k^{2} n^{2}-L n^{2} \\
& \left.\quad-L^{2} n^{2}\right)\left\langle r^{-(n+k+4)}\right\rangle+\left(k^{2} n-k L-k L^{2}-2 k n\right. \\
& \left.\quad-L n-L^{2} n+k n^{2}\right)\left\langle\beta V^{\prime}(r) r^{-(n+k+3)}\right\rangle \\
& \left.\quad+n k\left\langle\left[\beta V^{\prime}(r)\right]^{2} r^{-(n+k+2)}\right\rangle\right\} / M 0(L, n, k),
\end{aligned}
$$

where $\beta=1 / k_{\mathrm{B}} T, V^{\prime}(r)=[\mathrm{d} V(r)] / \mathrm{d} r$ and,

$$
\langle F(r)\rangle=4 \pi \int_{0}^{\infty} F(r) \exp (-\beta V(r)) r^{2} \mathrm{~d} r .
$$

In our spectrum we have seven different translational "subspectra" with different pairs of indices $L$ and $n+k$ as indicated in Fig. 4. The formulae for the translational moments $[27,29]$ require the isotropic potential between the $\mathrm{CO}_{2}$ molecules. We selected for this purpose a M3SV type potential.

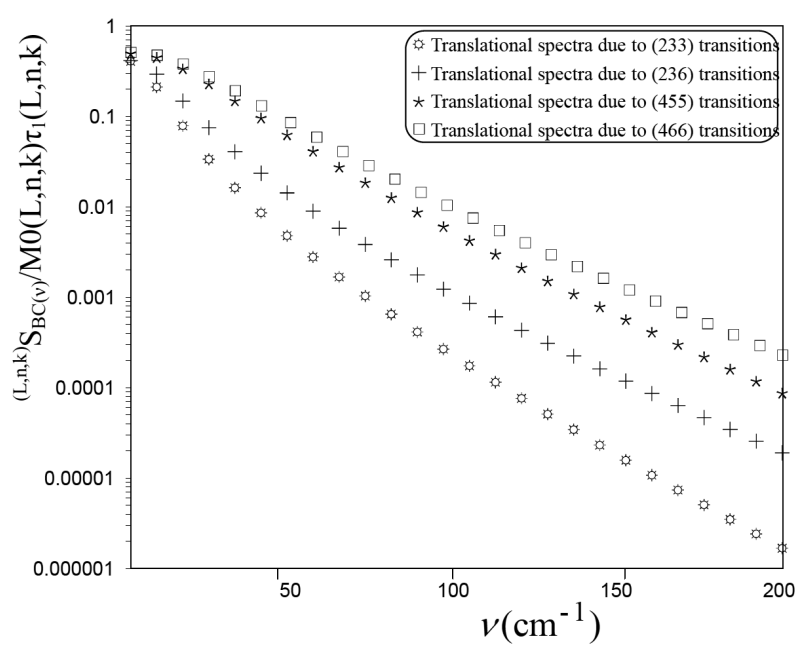

Fig. 4. Translational spectra for selected light scattering mechanisms of $\mathrm{CO}_{2}$ at $294.5 \mathrm{~K}$ as a function of frequency shifts. Calculations were performed with our new potential.

All spectral moments for light scattering mechanisms at room temperature $(T=294.5 \mathrm{~K})$ used in our computations, together with the characteristic times $\tau_{1}^{(L, n, k)}$ and $\tau_{2}^{(L, n, k)}$ of the respective Birnbaum-Cohen profiles are listed in Table II.

Finally, having calculated the rotational stick spectra and appropriate translational profiles, we compute with Eq. (11) our resulting spectrum as the convolution of the rotational and translational spectra.

\section{Results and discussion}

We have studied theoretically the anisotropic light scattering from gaseous carbon dioxide at $294.5 \mathrm{~K}$ and at density $1.026254 \mathrm{~mole} /$ litre. The results clearly show that, in all the spectra, the DID mechanism alone is not able to reproduce well their high-frequency interaction-induced rototranslational wings. To improve agreement between our theoretical spectra and the experimental ones we considered the multipolar light scattering mechanisms specified by Eq. (10). Figure 3 shows the comparison of the experimental anisotropic double differential cross-section and the theoretical cross-section. In the course of our calculations we found that the high frequency part of the anisotropic spectrum is mainly due to the components $\boldsymbol{B}_{4044}^{(2)}\left(R_{12}\right)=\boldsymbol{B}_{0444}^{(2)}\left(R_{12}\right)$ of the dipoleinduced octopole light scattering mechanism, which involves the dipole-octopole anisotropy tensor $\boldsymbol{E}_{4}$ and the isotropic part $\alpha$ of the linear polarizability. Contributions due to all the components give the intensities at relatively low frequencies and are significantly overshadowed by the permanent intrinsic anisotropy spectrum. 
TABLE II

The zeroth $M 0(L, n, k)$, and normalized second $\hat{M} 2(L, n, k)$, and fourth $\hat{M} 4(L, n, k)$ translational moments at $T=294.5 \mathrm{~K}$ for the successive light scattering mechanisms discussed in this work. The last two columns give the respective Birnbaum-Cohen profile characteristic times ${ }_{\tau_{1}}^{(L, n, k)}$ and ${ }_{\tau_{2}}^{(L, n, k)}$.

\begin{tabular}{|c|c|c|c|c|}
\hline$M 0(233)$ & $\hat{M} 2(233)$ & $\hat{M} 4(233)$ & $\begin{array}{l}(233) \\
\tau_{1}\end{array}$ & $\begin{array}{l}(233) \\
\tau_{2}\end{array}$ \\
\hline $\begin{array}{c}0.133 \AA^{-3} \\
M 0(236)\end{array}$ & $\begin{array}{c}7.71 \times 10^{24} \mathrm{~s}^{-2} \\
\hat{M} 2(236)\end{array}$ & $\begin{array}{c}6.86 \times 10^{50} \mathrm{~s}^{-4} \\
\hat{M} 4(236)\end{array}$ & $\begin{array}{c}6.069 \times 10^{-13} \mathrm{~s} \\
\underset{\tau_{1}}{(236)}\end{array}$ & $\begin{array}{c}2.14 \times 10^{-13} \mathrm{~s} \\
\underset{\tau_{2}}{(236)}\end{array}$ \\
\hline $\begin{array}{c}1.38 \times 10^{-3} \AA^{-6} \\
M 0(455)\end{array}$ & $\begin{array}{c}1.44 \times 10^{25} \mathrm{~s}^{-2} \\
\hat{M} 2(455)\end{array}$ & $\begin{array}{c}2.14 \times 10^{51} \mathrm{~s}^{-4} \\
\hat{M} 4(455)\end{array}$ & $\begin{array}{c}4.132 \times 10^{-13} \mathrm{~s} \\
\left(\begin{array}{c}(455) \\
\tau_{1}\end{array}\right.\end{array}$ & $\begin{array}{c}1.68 \times 10^{-13} \mathrm{~s} \\
\underset{\tau_{2}}{(455)}\end{array}$ \\
\hline $\begin{array}{c}3.19 \times 10^{-4} \AA^{-7} \\
M 0(456)\end{array}$ & $\begin{array}{c}2.77 \times 10^{25} \mathrm{~s}^{-2} \\
\hat{M} 2(456)\end{array}$ & $\begin{array}{c}4.97 \times 10^{51} \mathrm{~s}^{-4} \\
\hat{M} 4(456)\end{array}$ & $\begin{array}{c}2.045 \times 10^{-13} \mathrm{~s} \\
(456) \\
\tau_{1}\end{array}$ & $\begin{array}{c}1.76 \times 10^{-13} \mathrm{~s} \\
\underset{\tau_{2}}{(456)}\end{array}$ \\
\hline $\begin{array}{c}7.47 \times 10^{-5} \AA^{-8} \\
M 0(066)\end{array}$ & $\begin{array}{c}3.15 \times 10^{25} \mathrm{~s}^{-2} \\
\hat{M} 2(066)\end{array}$ & $\begin{array}{c}6.40 \times 10^{51} \mathrm{~s}^{-4} \\
\hat{M} 4(066)\end{array}$ & $\begin{array}{c}1.92 \times 10^{-13} \mathrm{~s} \\
\underset{\tau_{1}}{(066)}\end{array}$ & $\begin{array}{c}1.66 \times 10^{-13} \mathrm{~s} \\
\underset{\tau_{2}}{(066)}\end{array}$ \\
\hline $\begin{array}{c}1.77 \times 10^{-5} \AA^{-9} \\
M 0(266)\end{array}$ & $\begin{array}{c}2.31 \times 10^{25} \mathrm{~s}^{-2} \\
\hat{M} 2(266)\end{array}$ & $\begin{array}{c}5.43 \times 10^{51} \mathrm{~s}^{-4} \\
\hat{M} 4(266)\end{array}$ & $\begin{array}{c}3.22 \times 10^{-13} \mathrm{~s} \\
\underset{\tau_{1}}{(266)}\end{array}$ & $\begin{array}{c}1.34 \times 10^{-14} \mathrm{~s} \\
{ }_{\tau_{2}}^{(266)}\end{array}$ \\
\hline $\begin{array}{c}1.77 \times 10^{-5} \AA^{-9} \\
M 0(466)\end{array}$ & $\begin{array}{c}2.69 \times 10^{25} \mathrm{~s}^{-2} \\
\hat{M} 2(466)\end{array}$ & $\begin{array}{c}6.17 \times 10^{51} \mathrm{~s}^{-4} \\
\hat{M} 4(466)\end{array}$ & $2.61 \times 10^{-13} \mathrm{~s}$ & $\begin{array}{c}1.42 \times 10^{-14} \mathrm{~s} \\
\underset{\tau_{2}}{(466)}\end{array}$ \\
\hline $1.77 \times 10^{-5} \AA^{-9}$ & $3.59 \times 10^{25} \mathrm{~s}^{-2}$ & $8.25 \times 10^{51} \mathrm{~s}^{-4}$ & $1.78 \times 10^{-13} \mathrm{~s}$ & $1.57 \times 10^{-13} \mathrm{~s}$ \\
\hline
\end{tabular}

TABLE III

Numerical values of the carbon dioxide multipole polarizabilities and the permanent quadrupole moment used in our calculations (all values are in atomic units and from Refs. [30-35]).

\begin{tabular}{c|c|c|c|c|c|c|c|c}
\hline \hline$E_{x, x x x}$ & $E_{z, z z z}$ & $B_{x x, x x}$ & $B_{x x, z z}$ & $B_{z z, z z}$ & $B_{x z, x z}$ & $\Theta$ & $\alpha$ & $\gamma$ \\
\hline-64.0 & 175.4 & -125.0 & 65.0 & -195.0 & -156.0 & -3.201 & 17.93 & 1590
\end{tabular}

Our theoretical anisotropic double differential cross-sections were calculated using the numerical values of components of the carbon dioxide dipole-dipole $\alpha$, the dipole-octopole $\boldsymbol{E}_{4}$, the dipole-dipole-quadrupole $\boldsymbol{B}$ and the permanent quadrupole moment $\boldsymbol{\Theta}$ listed in Table III.

Quantum chemistry computations give $E_{4}=1.967 \AA^{5}$ $[30,31]$. Figure 3 shows however that the purely theoretical (based on the above mentioned computed values of the multipole polarizability tensors) anisotropic double differential scattering cross-sections give us too low intensity in comparison with the experimental values. The best fit of the experimental and theoretical spectra is obtained for $E_{4}=3.62 \AA^{5}$ as shown clearly in Fig. 5 .

The application of different broadening functions [6] leads to no significant difference in the final spectrum, obtained from first order information theory [36], the Mori theory, or the Birnbaum-Cohen [37] scheme. We thus conclude, in agreement with Barocchi et al. [38], that the profile of the rototranslational far wing is not sensitive to the details of the broadening function but reflects the distribution of the individual rotational lines of the spectrum.

One notices a distinct difference between the computed and fitted values of $E_{4}$. The discrepancy may suggest that there are other collisional mechanisms that should be considered in order to obtain a better theoretical description of the experimental spectrum. Nevertheless, we have shown that DIO provides an essential contribution to the wings, improving agreement between experimental and theoretical results. It is also worth noticing that the interaction-induced mechanism of $L=4$ symmetry with $E_{4}=3.62 \AA^{5}$ provides very good agreement, in the frequency range $0-115 \mathrm{~cm}^{-1}$, between the experimental and theoretical anisotropic rototranslational spectra of carbon dioxide. Beyond the $115 \mathrm{~cm}^{-1}$ frequency range some excess intensity appears. We attribute this intensity to a mechanism of shorter-range than DIO, such as an overlap mechanism, or the next higher multipole polarizability of $\mathrm{CO}_{2}$. When we model this contribution by an exponential curve $\exp \left(-\nu / \nu_{0}\right)$ with $\nu_{0}=115 \mathrm{~cm}^{-1}$, and add this contribution to the theoretical spectrum resulting from the permanent intrinsic anisotropy, DID and $\mathrm{DIO}+\mathrm{B} \Theta$ (with $E_{4}=3.62 \AA^{5}$ ) mechanisms, we obtain good agreement with the experimental results in the whole frequency range as shown in Fig. 5.

This conclusion is further supported by calculating the zeroth moment $M_{0}$ and the square-rooted reduced second moment $M_{2}^{-1 / 2}$ of the rototranslational spectrum.

$M_{0}$ is proportional to the total light scattering intensity and $M_{2}^{-1 / 2}$ is the band's mean extent over frequency and are given by 


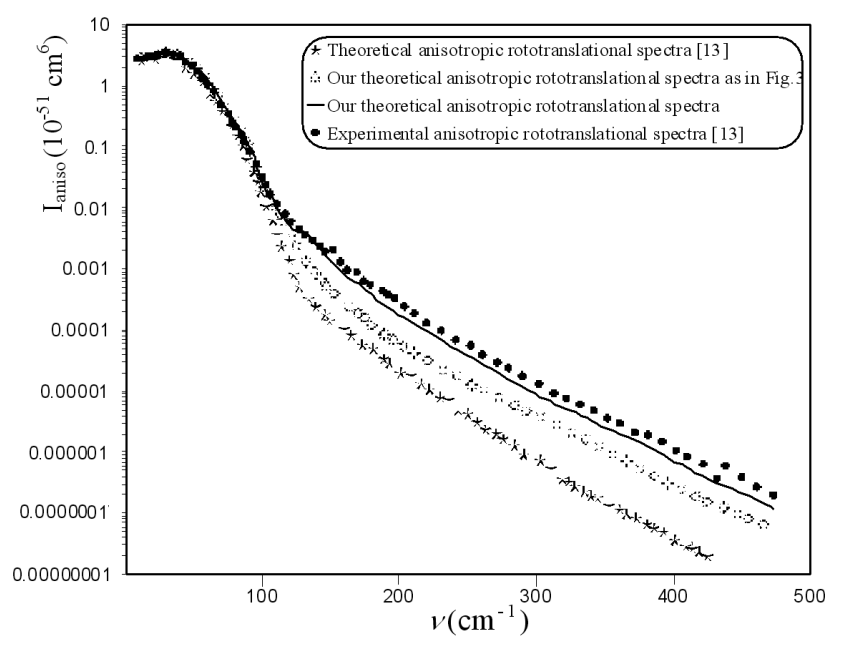

Fig. 5. The intensity of the rototranslational anisotropic spectra $\left(E_{4}=3.62 \AA^{5}\right)$ of $\mathrm{CO}_{2}$ at $294.5 \mathrm{~K}$ as a function of frequency shifts. The points refer to the experiment with the theoretical spectrum presented in [13]. Calculations were performed using M3SV potential with the parameters given in Table I and labeled 3. An exponential contribution $\exp \left(-\nu / \nu_{0}\right)$, with $\nu_{0}=115 \mathrm{~cm}^{-1}$, due to a very-short-range mechanism, has been included in the theoretical spectrum $(-)$.

$$
\begin{aligned}
& M_{2 n}=\frac{15}{2}\left(\frac{\lambda}{2 \pi}\right)^{4} \int_{-\infty}^{\infty}(2 \pi c \nu)^{2 n} I_{\text {aniso }}(\nu) \mathrm{d} \nu, \\
& M_{2}^{-1 / 2}=\left(\frac{1}{2 \pi C}\right) \sqrt{\frac{M_{2}}{M_{0}}}
\end{aligned}
$$

The experimental values for these moments are $0.08005 \pm$ $0.00651 \AA^{9}[39]$ and $(32 \pm 4.0) / 10^{8} \AA^{-1}[39]$ and the values calculated from Eqs. (24), (25) using our M3SV potential are $0.07815 \AA^{9}$ and $(30.4) / 10^{8} \AA^{-1}$.

\section{Summary}

Anisotropic rototranslational scattering from gaseous carbon dioxide in the far-frequency range up to about $500 \mathrm{~cm}^{-1}$ has been computed at room temperature 294.5 K and density about 1.026254 mole/litre using a new site-site intermolecular potential. This potential confirms that the thermodynamic and traditional transport properties over a wider temperature range can determine the interaction parameters. The experimental spectra have been compared with the theoretical ones calculated using a first and second-order DID light scattering, first order of the DIO as well as first order of the $\mathrm{B} \Theta$ light scattering mechanisms. Good agreement was found between the experimental and theoretical spectra in the frequency range up to $115 \mathrm{~cm}^{-1}$. When an exponential contribution with $\nu_{0}=115 \mathrm{~cm}^{-1}$ was considered to model the very short-range light scattering mechanisms at room temperature, good agreement between the theoretical and experimental spectra was found over the whole frequency range.

\section{References}

[1] D.P. Shelton, G.C. Tabisz, Mol. Phys. 40, 299 (1980).

[2] N. Meinander, A.R. Penner, U. Bafile, F. Barocchi, M. Zoppi, D.P. Shelton, G.C. Tabisz, Mol. Phys. 54, 493 (1985).

[3] M.S.A. El-Kader, Chem. Phys. 281, 49 (2002).

[4] U. Hohm, Chem. Phys. Lett. 379, 380 (2003).

[5] M.S.A. El-Kader, S.M. El-Sheikh, M. Omran, Z. Phys. Chem. 218, 1197 (2004).

[6] T. Bancewicz, V. Teboul, Y. Le Duff, Phys. Rev. A 46, 1349 (1992).

[7] Y. Le Duff, T. Bancewicz, W. Glaz, in: Collision and Interaction-Induced Spectroscopy, NATO ASI Series C: Mathematical and Physical Sciences Eds. G.C. Tabisz, M.N. Neuman, Kluwer Academic, Dordrecht 1994, p. 423.

[8] Y. Le Duff, R. Ouillon, J. Chem. Phys. 82, 1 (1985).

[9] U. Bafile, L. Ulivi, M. Zoppi, F. Barrochi, Phys. Rev. A 37, 1 (1988).

[10] M. Moraldi, A. Borysow, L. Frommhold, J. Chem. Phys. 88, 5344 (1988).

[11] M.S. Brown, S.K. Wang, L. Frommhold, Phys. Rev. A 40, 2276 (1989).

[12] L. Frommhold, J.D. Poli, R.H. Tipping, Phys. Rev. A 46, 2955 (1992).

[13] V. Teboul, Y. Le Duff, T. Bancewicz, J. Chem. Phys. 103, 1384 (1995).

[14] R.T. Pack, J.J. Valentini, C.H. Becker, R.J. Buss, Y.T. Lee, J. Chem. Phys. 77, 5475 (1982).

[15] F. Visser, P.E.S. Wormer, Chem. Phys. 92, 129 (1985).

[16] L. Monchick, K.S. Yun, E.A. Mason, J. Chem. Phys. 39, 654 (1963).

[17] E.W. Lemmon, M.O. McLinden, NIST Standard Reference Database 23: NIST Reference Fluid Thermodynamic and Transport Properties, Version 7.0 Beta, National Institute of Standards and Technology, Standard Reference Data Program, Gaithersburg 2001.

[18] A. Boushehri, J. Bzowski, J. Kestin, E.A. Mason, J. Phys. Chem. Ref. Data 16, 445 (1987).

[19] J.H. Dymond, E.B. Smith, The Virial Coefficients of Pure Gases and Mixtures, Oxford University, Oxford 1980.

[20] V. Vesovic, W.A. Wakeham, G.A. Olchowy, J.V. Sengers, J.T.R. Watson, J. Millat, J. Phys. Chem. Ref. Data 19, 763 (1990).

[21] M. Welker, G. Steinebrunner, J. Solca, H. Huber, Chem. Phys. 213, 253 (1996).

[22] S. Bock, E. Bich, E. Vogel, Chem. Phys. 257, 147 (2000).

[23] A.D. Buckingham, Adv. Chem. Phys. 12, 107 (1967).

[24] S. Kielich, Proc. Indian Acad. Sci. (Chem. Sci.) 94, 403 (1985).

[25] K.L.C. Hunt, Y.Q. Liang, S. Sethuraman, J. Chem. Phys. 89, 7126 (1988).

[26] T. Bancewicz, Mol. Phys. 50, 173 (1983).

[27] T. Bancewicz, V. Teboul, Y. Le Duff, Mol. Phys. 81, 1353 (1994). 
[28] W. Glaz, G.C. Tabisz, Can. J. Phys. 79, 801 (2001).

[29] T. Bancewicz, Chem. Phys. Lett. 213, 363 (1993).

[30] G. Maroulis, Chem. Phys. 291, 81 (2003).

[31] A. Haskopoulos, G. Maroulis, Chem. Phys. Lett. 417, 235 (2006).

[32] R.C. Burns, C. Graham, A.R.M. Weller, Mol. Phys. 59, 41 (1986).

[33] E. Stoll, Ann. Phys. 69, 81 (1922).

[34] D.P. Shelton, A.D. Buckingham, Phys. Rev. A 26, 2787 (1982)

[35] D.P. Shelton, J. Chem. Phys. 85, 4234 (1986).
[36] T. Bancewicz, W. Glaz, S. Kielich, Phys. Lett. A 148, 78 (1990).

[37] G. Birnbaum, E.R. Cohen, Can. J. Phys. 54, 593 (1976).

[38] F. Barocchi, A. Guasti, M. Zoppi, G.C. Tabisz, S.M. El-Sheikh, N. Meinander, Phys. Rev. A 39, 4537 (1989).

[39] M. Chrysos, A.P. Kouzov, N.I. Egorova, F. Rachet, Phys. Rev. Lett. 100, 133007 (2008). 